LINGÜÍSTICA 



\title{
LAS MARCAS LINGÜÍSTICAS DE OBLIGACIÓN EN ARTÍCULOS DE INVESTIGACIÓN EN TRES DISCIPLINAS ${ }^{1}$
}

\author{
Yosely Briceño Velazco
}

\begin{abstract}
RESUMEN
El artículo de investigación evidencia una variedad de fenómenos lingüísticos y discursivos, como la modalidad deóntica que se ocupa del 'deber ser'. Tomamos como base teórica los planteamientos de Thompson (1996), quien estudia la modulación, al igual que Halliday (1994). Nos propusimos estudiar el "deber ser" en Educación, Botánica e Ingeniería, con atención especial en las secciones de los artículos. Se tomó como unidades mayores de análisis el artículo de investigación y sus secciones. El corpus fue de 249.098 palabras. Se encontró variación en cuanto a: un $69.12 \%$ de las marcas lingüísticas de obligación se hallaron en Educación sobre un 26.17\%, en Ingeniería y 4.69\% de Botánica. En Educación, dichas marcas se concentran en la Introducción (38.25\%) y en los Resultados (17.11\%). En Botánica se concentran en la Introducción (2.01\%) y las Conclusiones $(1.34 \%)$. En Ingeniería en el Método (9.39\%) y también en la Introducción (6.71\%). Los hallazgos sugieren que las funciones de la obligación están determinadas por la disciplina y la sección del artículo donde aparecen.

Palabras clave: discurso disciplinar, discurso académico, artículos de investigación, modulación, evaluación.
\end{abstract}

\begin{abstract}
Research articles evidence a variety of linguistic and discourse phenomena such as deontic modality which has to do with 'must be'. As a theoretical background, proposals by Thompson (1996), who studies modulation, as well as Halliday's (1994) have been used. Our purpose was to analyze the 'must be' in education, botany and engineering with a special focus on article sections. The main units of analysis were the research article and its sections and the corpus was comprised of 249.098 words. Findings show variation in terms of a percentage of 69.12 linguistic marks of obligation found in education, $26.17 \%$ in engineering and 4.69 in botany. In education, such marks tend to appear in the Introduction (38.25\%) and in Results (17.11\%). In botany they are found mostly in the Introduction $(2.01 \%)$ and in Conclusions (1.34\%). In engineering, they appear in the Methods section $(9.39 \%)$ and also in the Introduction $(6.71 \%)$. These findings suggest that the function of obligation tends to be determined both by the specific discipline and the section of the article in which it appears.
\end{abstract}

Key words: disciplinary discourses, academic discourse, research article, modulation, evaluation.

\footnotetext{
Mgtr. Yosely Briceño Velazco. Profesora en la Escuela de Educación, Secretaria Académica de la Cátedra UNESCO para la Lectura y la Escritura, sub-sede Universidad Central de Venezuela.

Correo electrónico: yoselyj@gmail.com
}

Recepción: 18- 07- 2012

Aceptación: 10- 10- 2012 


\section{Introducción}

Algunos trabajos realizados han evidenciado la forma en que los investigadores, según la disciplina a la que pertenezcan, construyen y reportan el conocimiento (Bazerman 1988; Swales 1990, 2004; Hyland 2000; Bolívar 1997, 2004, 2006, 2011; Beke 2008; Beke y Bolívar 2009; Bolívar, Beke y Shiro 2010; Briceño 2010). Más aún, la manera como se construye en distintos géneros académicos (Hyland 2002; 2004; Ferrari 2003; Bolívar 2004, 2011; Swales 2004; Parodi 2005, 2006, 2007, 2010; Venegas 2006; Beke 2011; García Negroni 2008; Gutiérrez 2008; Beke y Bolívar 2009).

La escritura académica es reportada en gran medida mediante el género 'artículo de investigación'. Este género académico (Swales 1990, 2004) es uno de los que, por sus características discursivas particulares, puede reunir mayor evidencia linguiística sobre las actitudes, valores, creencias, grados de obligación y compromiso de quienes producen los textos. Por consiguiente, el artículo de investigación constituye un género académico evaluativo (Bolívar 2011), de ahí que se haya estudiado desde diferentes enfoques: el interaccional, el metadiscursivo (Chafe 1986; Hyland 2000, 2005; Bolívar 1997, 1999, 2005; Swales 2004), léxico-semántico (Venegas 2006) y desde la modalidad epistémica y deóntica (Bolívar 2001, 2006; Bolívar y Shiro 2005; Beke y Bolívar 2009; Bolívar, Beke y Shiro 2010) entre otros. En definitiva, el artículo de investigación es un género académico que reviste una gran importancia dentro de la dinámica de la investigación en las disciplinas.

Las investigaciones en el campo del discurso académico evaluativo (Hyland 2005; Ferrari y Gallardo 2006; Parodi 2006; Gutiérrez 2008; Bolívar 2008; Beke y Bolívar 2009; Tse y Hyland 2009) han mostrado que el estudio de la modalidad de obligación puede manifestar:

a) la manera como funcionan distintas comunidades discursivas y, b) que existen diferencias en la manera en la que cada disciplina construye discursivamente el conocimiento. Otros estudios (Bolívar 2001; Hyland 1999, 2000; Swales 1990) señalan que la elección entre una expresión lingüística y otra en el discurso académico se hace sobre la base de tradiciones discursivas ya existentes, y que estas tradiciones imponen un modo de escribir y de seleccionar modelos textuales (Bolívar 2005).

Si bien los estudios aludidos han dado cuenta, por una parte, del funcionamiento de las comunidades discursivas y de la forma como se construye el conocimiento en las disciplinas y; por otra parte, la manera cómo funcionan las tradiciones discursivas en la construcción de un género académico determinado, todavía no responden a aspectos más específicos, como por ejemplo, la actitud y el compromiso que asume quien escribe un artículo de investigación en cada sección del artículo de investigación, si existe variación lingüística y pragmática, cómo se dan entre disciplinas como la educación, la botánica y la ingeniería; y por último, qué implicaciones tiene todo ello para la escritura de artículos de investigación. Entonces, nos interesa centrar este estudio en revistas venezolanas y en disciplinas cuyas comunidades discursivas son bien diferenciadas (Swales 1990; Hyland 2000; Bolívar 2001, 2005; Parodi 2006; Beke y Bolívar 2009; Gutiérrez 2008).

El presente estudio podría ser doblemente revelador. Por un lado, nos permitiría conocer características muy particulares de la modalidad de obligación en tres disciplinas distintas. Por otro, arrojaría luces en cuanto a las actitudes y concepciones sobre investigación que puedan tener los investigadores según la disciplina a la que pertenezcan. Por lo tanto, el objetivo principal de este estudio es analizar un corpus de artículos de investigación de educación, botánica e 
ingeniería con el propósito de averiguar cómo se manifiesta lingüística y discursivamente la modalidad de obligación en estas tres disciplinas y, también, cómo varía su función según la sección del artículo en el que aparecen.

\section{La modalidad deóntica como "modulación"}

En los últimos años, los estudios sobre la evaluación han sido frecuentes en el campo del discurso en general (Bolívar 2001, 2005; Hunston 2001, Hunston y Thompson 2000), y en el discurso académico y científico en particular (Hunston 1994, 2001; Bolívar 2004, 2006; Harvey 2005). Especialmente, se tienen estudios con artículos de investigación en los que se analizan aspectos de la evaluación, como los que se mencionaron más arriba (Chafe 1986; Hyland 2000, 2005; Bolívar 1997, 1999, 2001, 2005, 2006; Swales 2004; Venegas 2006).

La mayor parte de los estudios sobre evaluación académica se han hecho de acuerdo con la metodología de la lingüística dirigida por corpus (Hunston 2002; Sinclair 2003, 2004), que se combina con el análisis de textos particulares. La combinación de ambas metodologías nos permitiría llegar a una mejor comprensión sobre el fenómeno que se está estudiando (Biber, Conrad y Reppen 1998; Beke 2011; Bolívar 2011).

En este estudio nos concentraremos en aquella evaluación que tiene que ver con el grado de obligación y de compromiso modal de quien escribe un artículo de investigación. Para ello, partimos de Thompson, quien, siguiendo el modelo de Halliday (1985), estudia la modalidad deóntica con el nombre de 'modulación' y distingue la 'obligación', refiriéndose a ésta como el grado de obligación con el otro (1996: 57-58). El emisor, según la forma que emplee para expresar la modalidad, puede asumir mayor o menor "compromiso modal". Este término se aplica al grado en que el hablante se compromete con la certeza de sus afirmaciones o con la presión que ejerce para imponer su punto de vista (Thompson 1996). La modalidad de obligación como evaluación en la escritura académica cumple una función muy importante, por dos razones principalmente. Por un lado, porque desde el punto de vista de la investigación, quien escribe está en la capacidad de promover cambios deseables para el bien de la comunidad discursiva y de la disciplina. Por otro, porque suponemos que quien escribe es un agente moralmente responsable para ejercer presión sobre el otro para que lleve a cabo los cambios deseables para el fortalecimiento de la disciplina (Bolívar 2004). En este juego, las tradiciones discursivas se construyen, se fortalecen y se mantienen en el tiempo.

Por otra parte, algunos estudios han dado cuenta de la variedad lingüística con la que puede expresarse la modalidad de obligación. Ente ellas se encuentran los sufijos, los clíticos y los participios; las inflexiones y los verbos modales (Palmer 2001); el adjetivo, los verbos modales y el adverbio (Hyland 2000, 2005; Martín y White 2005); los adjetivos predicativos que expresan el juicio del autor en cuanto a la necesidad de o la importancia de (es importante que, es necesario que) (Hyland 2005); los verbos modales (poder, deber); adjetivos y adverbios de necesidad (necesario, necesariamente) y el uso de la primera persona en determinados contextos, como en la realización del movimiento denominado "ocupando el nicho" en la Introducción de los artículos (Swales 1990: 140). Otros estudios en los que se han comparado disciplinas humanísticas y científicas en los artículos de investigación han encontrado que la modalidad de obligación puede manifestarse en los modales debe, debería, tiene que, y en adjetivos predicativos como (es) importante que, (es) necesario que (Hyland 2005); verbos de obligación (se requiere, se recomienda) y de adjetivos con valor deóntico (es) necesario, (es) 
indispensable (Ferrari y Gallardo 2006); léxico valorativo, verbos modales (debe, debería y hay que), con adverbios, con directivos indirectos en cláusulas condicionales y con cláusulas subordinadas con subjuntivo (Bolívar 2006; Beke y Bolívar 2009). En este trabajo analizaremos los verbos modales y otras formas verbales, además de otras estructuras lingüísticas como adjetivos y adverbios que expresen la modalidad de obligación, porque nos interesa conocer el tipo de estructura y la función discursiva en la sección del artículo de investigación en la que se manifiesten en las tres disciplinas estudiadas.

\section{Las funciones retóricas de las secciones del artículo de investigación}

El creciente aumento de trabajos en los que se ha analizado al artículo de investigación desde diferentes perspectivas (Hyland 2000; Swales 1990, 2004; Thompson 1996; Ferrari 2003; Ferrari y Gallardo 2006; Venegas 2006; Beke y Bolívar 2009; Bolívar Beke y Shiro 2010) ha permitido conocer, por un lado, la caracterización de este género académico; y por otro, cada una de las secciones que lo estructuran. Este tipo de texto ha sido uno de los géneros que más se ha venido estudiando, probablemente porque en él la evaluación se puede codificar de diferentes maneras y además el foco de análisis puede variar según el problema planteado y los objetivos de la investigación (Bolívar 2006). En este trabajo nos concentramos en el artículo de investigación que reporta una investigación empírica culminada, el cual tiende a adoptar en un nivel superficial divisiones estandarizadas o sus variantes según las disciplinas, tal como la de IMRD (Introducción, Método, Resultados y Discusión) (Swales 1990: 175). Esto debido a que nos interesa examinar las funciones del "deber ser" en cada una de las secciones del artículo de investigación.

Los estudios en los que se han examinados las secciones del artículo de investigación de carácter empírico han sido muy relevantes. Así, por ejemplo, en la sección de la Introducción (Swales 2004; Samraj 2002; Ferrari y Gallardo 2006; Bolívar, Beke y Shiro 2010; Bolívar y Bolet 2011) se puede observar la cantidad y el tipo del conocimiento establecido, la postura por asumir, lo directo o indirecto del enfoque, el uso de la persuasión y el manejo de la relativa atractividad del mensaje para el lector, entre otros rasgos, en las marcas de evaluación que proporciona y en la forma como se estructura el texto. Adicionalmente, Bathia (1993) señala que la característica de la Introducción como género depende del propósito comunicativo que pretende cumplir. En tal sentido Hyland (2000: 91) señala que el mayor número de recursos mitigadores y resaltadores aparecen en las "Introducciones" y las "Conclusiones", en consonancia con la afirmación de Bazerman (1988: 243) de que son lo que los físicos leen primero en una lectura rápida, para juzgar el valor del artículo para su propia investigación. Por su parte, en la sección del Método, Swales $(1990,2004)$ señala que en esta sección se incluye la descripción, incluso deficiente, del método aplicado y, al igual que en la introducción, el recurso a fuentes bibliográficas. No obstante, esto puede variar de una disciplina a otra (Briceño 2010).

En cuanto a la sección de los Resultados, sostiene Swales (1990, 2004) que la mayoría de los artículos de investigación tienen secciones de resultados y discusión como cierre, pero que en otros se mezclan ambos, mientras que otros utilizan denominaciones como Conclusiones, Implicaciones, Aplicaciones, y así por el estilo, de modo que puede haber una combinación de estos aspectos. Más aún, señala que, desde el punto de vista del analista del discurso, hay bastante variación en el grado en el cual la sección de resultados simplemente 
describe los resultados y la de discusión los discute (Swales 1990: 171-172). En cuanto a la sección de Conclusiones, señalan varios autores (Hyland 2000: 67; Paltridge 1994: 291; Swales 2004: 235) que esta es una sección que podría implicar variabilidad dentro del género del artículo de investigación, denominándose en algunos casos "discusión". En ella se presentan normalmente interpretaciones, reflexiones e inferencias con las cuales el autor podría extender los resultados fuera y más allá del estudio en particular. Ferrari (2005) y Ferrari y Gallardo (2006), al igual que los autores anglosajones señalados, concluyen que de las cuatro secciones del artículo de investigación, es en la 'Conclusiones' (también señalan la Introducción) donde mayormente aparecen rasgos de la modalidad, así como de otros recursos de la evaluación.

Como podrá verse, las secciones del artículo de investigación reflejan un tipo de información específica. Bolívar (2000) y Venegas (2006) han sostenido que la estructuración del artículo de investigación bajo el patrón genérico arriba descrito podría representar la utilización de una doble estrategia para el investigador. Por un lado, elaborar un artículo de investigación "bien estructurado", ajustado a las normas de publicación requeridas por gran parte de las revistas en el ámbito nacional e internacional y, por otro, persuadir a una comunidad discursiva de nivel internacional para lograr su acceso a ella.

\section{El método, el corpus y los procedimientos}

Este estudio es de tipo cuantitativo-cualitativo. En él se combinan técnicas de análisis computarizado basado en la lingüística de corpus (Stubbs 1996, 2006; Simpson y Swales 2001; Bolívar 2006; Parodi 2006; Beke y Bolívar 2009; Bolívar 2011), y el análisis focalizado en interacción con los textos (Bolívar 2006). Ambos análisis se aplicaron para conocer las posibles diferencias entre las disciplinas (educación, botánica e ingeniería) en cuanto al uso del "deber ser" y a la sección del artículo en el que aparece. Para el análisis se utilizaron las formas verbales (deba, deban, debe, debemos, deberá, deberán, debería, deberían, debía, debieran, debo, ha de, han de, hay que, hemos de, precisa, preciso, requerirá, requiere, requieren, tendrá, tendrán, tendría, tendrían, tenemos), sustantivos o grupos nominales (la necesidad de, la obligación de), adjetivos (conveniente, imprescindible, necesario, necesaria), adverbios (necesariamente, obligatoriamente, obligadamente, definitivamente, absolutamente). Como unidades mayores de análisis se tomó el texto artículo de investigación y sus secciones (Introducción, Método, Resultados, Conclusiones o Discusión).

El corpus consistió de 37 artículos de investigación ${ }^{2}$, a saber: 13 artículos de Educación, 14 de Botánica y 10 de Ingeniería. Todos publicados en el año 2007³. Los 37 artículos sumaron 249.098 palabras en total ${ }^{4}$. Cada artículo se guardó en su totalidad en formato de Texto (txt). A cada artículo se le asignó un código, siguiendo los criterios empleados por Beke (2011) y ampliados en Briceño (2010), en el que se indicaron el volumen y número de la revista, el año de publicación, un dígito para diferenciar cada artículo y la inicial del nombre de la disciplina. Así, por ejemplo 28810701-E correspondió al primer artículo del corpus del volumen 28, número 81 del año 2007 de la Revista de Pedagogía, de Educación, y así por el estilo.

La tabla 1 muestra los textos del corpus, el código empleado para su búsqueda y el número de palabras. El promedio de palabras por texto fue de 7.000. Se observó variación por disciplina, ya que los textos de Educación promediaron las 7.000 mil palabras, mientras que 
en Botánica había de 20.000 mil y otros con solo 700 palabras. Por su parte, en Ingeniería la extensión iba desde las 2.000 mil hasta las 10.000 mil palabras.

Tabla 1. El corpus

\begin{tabular}{|c|c|c|c|c|c|}
\hline $\begin{array}{c}\mathrm{N}^{0} \text { de } \\
\text { artículo }\end{array}$ & Código & $\mathbf{N}^{o}$ de palabras & $\begin{array}{c}\mathbf{N}^{0} \text { de } \\
\text { artículo }\end{array}$ & Código & $\mathrm{N}^{0}$ de palabras \\
\hline 1 & 28810701-E & 5.922 & 20 & 30010720-B & 3.090 \\
\hline 2 & 28810702-E & 8.155 & 21 & 30010721-B & 719 \\
\hline 3 & 28810703-E & 6.133 & 22 & 30020722-B & 1.566 \\
\hline 4 & 28810704-E & 5.053 & 23 & 30020723-B & 7.388 \\
\hline 5 & $28820705-\mathrm{E}$ & 6.412 & 24 & 30020724-B & 1.295 \\
\hline 6 & 28820706-E & 7.676 & 25 & 30020725-B & 870 \\
\hline 7 & 28820707-E & 8.004 & 26 & 30020726-B & 4.151 \\
\hline 8 & 28820708-E & 7.487 & 27 & 30020727-B & 8.581 \\
\hline 9 & 28820709-E & 7.201 & 28 & 45010728-I & 7.121 \\
\hline 10 & 28830710-E & 5.137 & 29 & 45010729-I & 5.463 \\
\hline 11 & 28830711-E & 10.014 & 30 & 45010730-I & 4.080 \\
\hline 12 & 28830712-E & 9.148 & 31 & 45020731-I & 2.776 \\
\hline 13 & 28830713-E & 7.523 & 32 & 45020732-I & 6.157 \\
\hline 14 & 30010714-B & 4.575 & 33 & 45020733-I & 7.631 \\
\hline 15 & 30010715-B & 5.471 & 34 & 45020734-I & 4.682 \\
\hline 16 & 30010716-B & 25.201 & 35 & 45030735-I & 2.554 \\
\hline 17 & 30010717-B & 20.646 & 36 & 45030736-I & 10.187 \\
\hline 18 & 30010718-B & 8.774 & 37 & 45030737-I & 4.541 \\
\hline 19 & 30010719-B & 7.714 & \multicolumn{2}{|c|}{ Total } & 249.098 \\
\hline
\end{tabular}

Se realizó la búsqueda de las marcas linguiísticas de obligación con el programa WordPilot 2000. Se descartaron todas aquellas señales que no reflejaran esta modalidad, como por ejemplo, el modal 'debe' en función de modalidad epistémica, como se muestra en el siguiente ejemplo, tomado de un texto de Ingeniería:

(1) Si los padres al cruzarse están muy cercanos, la probabilidad de mutación a aplicar a los hijos debería ser alta.

En la Tabla 2 se observan todas las señales lingüísticas del 'deber ser' encontradas, y la sección del artículo donde aparecen. Nótese la diferencia entre los valores relativos más altos de "deber", "ser" y "requerir", comparados con "tener que". Por otra parte, obsérvese que de acuerdo con el análisis de los datos, el número de marcas de obligación es más alto en la sección de la Introducción, con un 53.35\%, seguido de la sección de Resultados con 25.83\%, mientras que la sección del Método alcanzó un 15.12\%. La sección de Conclusiones alcanzó un 10.33\%. 
Tabla 2. Total de marcas lingüísticas de obligación por sección del artículo de investigación

\begin{tabular}{|c|c|c|c|c|c|}
\hline \multirow{2}{*}{$\begin{array}{c}\text { Marcas lingüísticas } \\
\text { del 'deber ser" }\end{array}$} & \multicolumn{3}{|c|}{ Sección del artículo de investigación } & $\begin{array}{c}\text { Total de marcas lingüísticas } \\
\text { del "deber ser" en los } \\
\text { artículos de investigación }\end{array}$ \\
\cline { 2 - 5 } & I & M & R & C & 151 \\
\hline 'deber' & 99 & 14 & 30 & 8 & 33 \\
'requerir' & 16 & 7 & 7 & 3 & 32 \\
'ser' & 15 & 6 & 7 & 4 & 29 \\
'la necesidad de' & 7 & 5 & 12 & 5 & 28 \\
'necesario/a de' & 10 & 5 & 6 & 7 & 15 \\
'haber' & 4 & 3 & 7 & 1 & 4 \\
'la obligación de' & 4 & 0 & 0 & 0 & 3 \\
'precisa de' & 3 & 0 & 0 & 0 & 3 \\
'tener que' & 1 & 1 & 1 & 0 & 298 \\
\hline Total de marcas & 159 & 41 & 70 & 28 & $(100 \%)$ \\
lingüísticas del & $(53.35 \%)$ & $(15.12 \%)$ & $(25.83 \%)$ & $(10.33 \%)$ & \\
"deber ser" por & & & & & \\
sección del artículo & & & & & \\
\hline
\end{tabular}

A fin de averiguar acerca del uso del 'deber ser' en las tres disciplinas, en el contexto de la sección del artículo donde aparecían, se examinaron las concordancias de todas las estructuras lingüísticas mostradas en la Tabla 2. Ello debido a que nos interesaba concentrar nuestro análisis en la función discursiva que cumplían estas marcas en cada sección del artículo. El análisis se realizó en interacción con los textos (Bolívar 2006) examinando cada uno de los casos en su co-texto, es decir, la sección del artículo, y en el contexto del artículo completo.

\section{Resultados}

\subsection{Las funciones discursivas del "deber ser" en las disciplinas}

Del análisis cuantitativo se obtuvo que, aproximadamente, en Educación se emplea la modalidad de obligación tres veces más que en Ingeniería y trece veces más que en Botánica. Si se comparan Ingeniería y Botánica, se utiliza cuatro veces más la modalidad de obligación en la primera que en la segunda, tal como puede observarse en el cuadro 1.

Cuadro 1. Total de palabras, número de marcas de obligación y frecuencia por cada mil palabras en las disciplinas

\begin{tabular}{|c|c|c|c|}
\hline & Educación & Botánica & Ingeniería \\
\hline Total de palabras & 93.865 & 100.041 & 55.192 \\
Total de marcas & 206 & 14 & 78 \\
Marcas por mil palabras & 2.19 & 0.13 & 1.41 \\
\hline
\end{tabular}

En cuanto a la frecuencia relativa, por cada mil palabras se tiene que para un corpus de 249.098 hay una frecuencia de la modalidad de obligación de 1.19 por cada mil palabras. La variación entre Educación e Ingeniería es de 0.78 por mil palabras, mientras que entre Educación y Botánica es de 2.06. Se deduce del cuadro 1 que la modalidad de obligación no es una característica representativa de disciplinas como la Botánica. 


\subsubsection{Los tipos de estructura lingüística de obligación por disciplina y sección del artículo}

Nótese en la tabla 3 más abajo que la estructura verbal para reportar la obligación aparece con frecuencia de un $80.5 \%$, las nominales $11.07 \%$ y las adjetivales $8.38 \%$. Por otra parte, en el análisis por disciplina se encontró que en Educación, de 206 marcas el 77.66\% de las estructuras lingüísticas de obligación son verbales, $11.65 \%$ son nominales y $10.67 \%$ adjetivales. Este hallazgo coincide con el estudio realizado por Beke y Bolívar (2009). En cuanto a Botánica, de 14 marcas el $71.42 \%$ son estructuras verbales y $28.57 \%$ nominales. En Ingeniería de 78 marcas de obligación, $89.74 \%$ lo constituyen las estructuras verbales, $6.41 \%$ las nominales y $3.84 \%$ las adjetivales. Si se comparan estas tres disciplinas, es en Ingeniería en la cual el mayor número de marcas lingüísticas de obligación es de tipo verbal.

Tabla 3. Tipos de estructuras lingüísticas de obligación en cada disciplina

\begin{tabular}{|c|c|c|c|c|c|}
\hline \multirow[b]{2}{*}{ MARCA LINGÜÍSTICA } & \multicolumn{3}{|c|}{ DISCIPLINAS } & \multirow{2}{*}{$\begin{array}{l}\text { Número } \\
\text { total de } \\
\text { marcas } \\
\text { linguiísticas }\end{array}$} & \multirow{2}{*}{$\begin{array}{l}\text { Número total } \\
\text { de marcas } \\
\text { lingüísticas } \\
\text { por tipo de } \\
\text { estructura }\end{array}$} \\
\hline & EDUCACIÓN & BOTÁNICA & INGENIERÍA & & \\
\hline $\begin{array}{l}\text { ESTRUCTURAS } \\
\text { VERBALES: } \\
\text { 'DEBER' } \\
\text { 'REQUERIR' } \\
\text { 'SER' } \\
\text { 'HABER' } \\
\text { 'PRECISA DE' } \\
\text { 'TENER QUE' } \\
\end{array}$ & $\begin{array}{c}101 \\
21 \\
19 \\
14 \\
3 \\
2\end{array}$ & $\begin{array}{l}1 \\
8 \\
1 \\
0 \\
0 \\
0\end{array}$ & $\begin{array}{c}42 \\
17 \\
8 \\
2 \\
0 \\
1\end{array}$ & $\begin{array}{c}144 \\
46 \\
28 \\
16 \\
3 \\
3\end{array}$ & $\begin{array}{c}240 \\
80.5 \%\end{array}$ \\
\hline $\begin{array}{c}\text { ESTRUCTURAS } \\
\text { NOMINALES: } \\
\text { 'LA NECESIDAD DE' } \\
\text { 'LA OBLIGACIÓN DE' }\end{array}$ & $\begin{array}{c}21 \\
3\end{array}$ & $\begin{array}{l}3 \\
1\end{array}$ & $\begin{array}{l}5 \\
0\end{array}$ & $\begin{array}{c}29 \\
4\end{array}$ & $\begin{array}{c}33 \\
11.07 \%\end{array}$ \\
\hline $\begin{array}{c}\text { ESTRUCTURAS } \\
\text { ADJETIVALES: } \\
\text { 'NECESARIO/A DE' }\end{array}$ & 22 & 0 & 3 & 25 & $\begin{array}{c}25 \\
8.38 \%\end{array}$ \\
\hline $\begin{array}{c}\text { Número total de marcas } \\
\text { de obligación en cada } \\
\text { disciplina }\end{array}$ & 206 & 14 & 78 & 298 & \\
\hline
\end{tabular}

Adicionalmente, al examinar estas estructuras lingüísticas según la sección del artículo y la disciplina se encontró lo siguiente:

Tabla 4. Total de estructuras lingüísticas de obligación por sección del artículo en las disciplinas

\begin{tabular}{|c|c|c|c|c|}
\hline \multirow{2}{*}{$\begin{array}{c}\text { SECCIÓN DEL } \\
\text { ARTÍCULO }\end{array}$} & \multicolumn{3}{|c|}{ DISCIPLINAS } & $\begin{array}{c}\text { Total de número } \\
\text { de marcas por sección del artículo }\end{array}$ \\
\hline Introducción & 114 & 6 & 20 & $140(46.97 \%)$ \\
\hline Método & 11 & 2 & 28 & $41(13.75 \%)$ \\
\hline Resultados & 51 & 2 & 18 & $71(23.82 \%)$ \\
\hline Conclusiones & 30 & 4 & 12 & $46(15.43 \%)$ \\
\hline
\end{tabular}


Obsérvese en la tabla 4 que en Educación las marcas de obligación se concentran en la Introducción (114 marcas) y en los Resultados (51 marcas). En Botánica, similarmente a Educación las marcas de obligación se concentran en la Introducción (6 marcas), pero también en las Conclusiones (4 marcas). En Ingeniería, aparecen mayormente en la sección de Método (28 marcas) y de Introducción (20 marcas). Sostenemos que en Ingeniería, por tratarse de una disciplina muy técnica, los investigadores se preocupan más por señalar recomendaciones y acciones que deben llevarse a cabo de una determinada manera, y esto se refleja en los procedimientos y en las técnicas a emplearse. En todo caso, las diferencias en cuanto al empleo de las marcas de obligación en las disciplinas no solo parecieran deberse a la tradición discursiva de esta (Bolívar 2004), sino también a factores relacionados con la estructuración del artículo de investigación.

\subsubsection{La función del deber ser ante la materia, la investigación y la actitud con la disciplina}

Del análisis focalizado se observó que existe variación, por un lado, en cuanto al tipo de marca lingüística, y por otro, en relación con el tipo de obligación reportada. Esa variación viene dada por el tipo de acción deseable o no deseable que en cada una de las secciones del artículo de investigación se reporta, tal como veremos de aquí en adelante.

\subsubsection{En la sección de la introducción: lo que la materia debe ser}

Aquí el uso del "deber ser" está asociado con la actitud ante el contenido de la materia o la disciplina por medio del uso de verbos como "debe", "requerir de", sser" y "tener que"; adjetivos como "necesario/ria de" y nominalizaciones como "la necesidad de", "la obligación de”. Los cambios que se promueven están relacionados con:

a. La actuación del docente en el aula de clase

(2) Durante el trabajo en el aula con los y las estudiantes, es posible que el docente deba adaptar, improvisar y experimentar nuevas estrategias, relacionar el tema $\underline{\text { tratado }}$ con otros distintos y desconocidos, tomar decisiones para controlar la dinámica del grupo y conducirlo hacia el logro de los objetivos. Por ello, durante la clase el docente debe mantenerse en un estado de alerta que le permita incorporar nuevos aprendizajes a su formación. (28820709-E)

b. El proceso de la investigación para construir conocimientos

(3) Es necesario comprender que la sistematización es un proceso de investigación que orienta la construcción de conocimientos situacionales producto de los acontecimientos concretos que, no necesariamente deban ser generalizados. (28820705-E)

(4) Esta respuesta es posible debido a la continua producción y elongación de nuevos entrenudos, para lo cual requiere de carbohidratos adicionales que pueden provenir de la redistribución de los recursos desde las hojas senescentes o desde los órganos subterráneos hacia hojas nuevas. (30010714-B) 
(5) Se ha demostrado que los efectos torsionales elásticos deben evaluarse en términos de sus efectos en las líneas o planos resistentes, fundamentalmente en los extremos, donde son más desfavorables [Dempsey y Tso, 1982; Hernández y López, 1984], y que denominaremos críticos. Esta necesidad se debe principalmente a la no simultaneidad de los respectivos valores máximos de los cortantes de piso y momentos torsores, la cual sería necesario considerar para estimar con precisión la demanda en el extremo flexible de las plantas y conduciría al requerimiento de otro valor y otro signo del momento torsor, para estimar la demanda en el extremo rígido de las plantas. (45030736-I)

Como podrá notarse en los ejemplos anteriores $(2,3,4,5)$, existe un desarrollo retórico en los enunciados que da evidencia de que se introduce al lector en el tópico de la investigación, y en los que hemos subrayado las palabras que ayudan a evidenciar este hecho (Swales 2004). Adicionalmente, tenemos el uso de los verbos frecuentemente en tiempo presente y el uso de la voz pasiva (Swales 2004). Nótese que tanto en Educación y en Ingeniería se utiliza más el modal 'debe', y en Botánica el verbo usualmente evidenciado es 'requiere'. Inferimos que estas diferencias están asociadas con el objeto de estudio, porque cuando se emplea el verbo 'requiere' en Educación (como se muestra en el ejemplo 6) es para hacer alusión a una necesidad que no tiene alternativa de que pueda darse de otra manera.

(6) El cuerpo docente y directivo requiere estudiar la población que asiste a la escuela, conocer a los alumnos y sus representantes, sus necesidades [...]. (28820708-E)

Por su parte, en el caso de Botánica, la necesidad está referida a componentes esenciales para la subsistencia de las plantas (ejemplo 4). Esto último ocurre en Ingeniería cuando el requerimiento se hace para la ejecución de un procedimiento propio de dicha disciplina. Por otro lado, también puede verse en los casos arriba señalados la predominancia de léxico que indica información relacionada con el contenido de la materia, como por ejemplo, en Educación estrategias, didáctica, grupo, sistematización, conocimiento, valores; en Botánica entrenudos, carbohidratos, hojas; y en Ingeniería efectos torsionales, valores, cortantes de piso. En definitiva, en Educación la obligación tiene su foco en promover cambios favorables en la actuación del docente en el aula y en los no deseables, como lo son la generalización apresurada en cuanto a las estrategias de investigación del docente para construir nuevos conocimientos. En Botánica e Ingeniería la obligación se centra en aspectos propios de la materia y que están directamente relacionados con la investigación.

5.1.2.2. En la sección del Método: ¿cómo debe ser la investigación? ¿qué se debe hacer?

En cuanto a esta sección, se emplean verbos como 'debe', 'requerir de', 'ser' y 'tener que' y la nominalización 'la necesidad de'. Adicionalmente, se encontró que la obligación en esta sección del artículo busca promover cambios deseables en cuanto a:

a. Cómo deben ser los materiales para elaborar materiales didácticos

(7) Con respecto a los materiales, es necesario que sean de buena calidad, porque es preferible que los niños y familiares elaboren álbumes que puedan 
manejarse con facilidad y seguir agregándole hojas y dibujos realizados por ellos mismos. (28830712-E)

b. Cómo debe llevarse el proceso de la investigación misma

(8) Sobre este particular se debe mencionar que repetidamente hubo inconsistencias entre lo que se indicaba sobre la colección y la información que aparecía en la publicación original. Adicionalmente, la consulta de fuentes bibliográficas originales permitió extraer un gran número de tipos intercalados en la colección general o en espera de ser montados, así como identificar los herbarios nacionales o extranjeros donde se encuentra el resto de los tipos (holótipos o isótipos) asociados al taxon. (30010716-B)

(9) Estructuras más complejas que las figuras geométricas clásicas requieren una metodología para ser representadas como grupos de células discretas. Para el modelado de las prótesis mecánicas de válvulas aórticas se consideró el uso de herramientas CAD para su representación geométrica en programas computacionales. (45010728-I)

Como puede verse en los ejemplos anteriores $(7,8,9)$, la modalidad de obligación en la sección del método está referida a aspectos concretos, es decir, que desde el punto de vista funcional la imposición se relaciona con procesos materiales. Por su parte, en Educación la preponderancia del verbo 'debe' y de estructuras con 'ser' hace pensar que no es un simple deseo de que se lleve a cabo el evento. Ello refleja menos flexibilidad en el momento de ejercer presión sobre el otro y sobre sí mismo en lo que a procedimientos e instrumentos de la investigación se refiere. Los cambios que se promueven tienen que ver con los componentes que se emplean para elaborar materiales para el trabajo de aula. En Botánica, la obligación se asocia con la necesidad de reportar inconvenientes en la aplicación de la metodología para llevar a cabo el estudio. Se infiere que esta sección, para disciplinas como ésta, es muy importante, y que cualquier error o dificultad durante su aplicación deberá ser notificada para evitar procedimientos no deseables en un futuro. En Ingeniería, el foco de la obligación, al igual como ocurre en Botánica, se centra en los procedimientos y en el método. En definitiva, la actitud del investigador en esta sección del artículo está asociada con el proceso de la investigación, nótese el léxico empleado, como por ejemplo, materiales, elaborar, realizar, consultar, fuentes, extraer, identificar, considerar, herramientas.

\subsubsection{En la sección de Resultados: ¿qué debe ser?}

En la sección de Resultados, al igual que en el resto de las otras dos secciones, los verbos, y en especial el modal 'debe', son los más recurrentes. Por otra parte, la modalidad de obligación en esta sección del artículo se relaciona a aspectos tales como:

a. Cómo debe ser el proceso de investigación para orientar las acciones en el docente.

(10) El ciclo demostró la necesidad de orientar las acciones hacia implementar estrategias que promuevan valores y acciones para enfrentar la violencia, con sus manifestaciones de injusticia, tanto en el aula como en la escuela en general. (28820705-E) 
b. Cómo debe ser el proceso de investigación para registrar las necesidades dentro del aula de clases.

(11) Otras necesidades que se extrajeron del diario de campo llevado durante varias reuniones fueron: revisar las estrategias de enseñanza: hay que manejar mayor número de estrategias porque si los estudiantes no entienden una, hay que buscar otra, no repetir la misma. (28820709-E)

c. Cómo debe ser el proceso de investigación para estimar la producción de semillas en las plantas.

(12) Además, es conveniente considerar que el número de semillas por fruto representa un mejor estimado reproductivo, ya que los frutos pueden variar ampliamente en el número de semillas por fruto, lo cual puede responder entre muchas variables a relaciones de incompatibilidad. (30020730-B)

d. Lo que se debe atribuir en el proceso de investigación

(13) En todos los hormigones (HC y HR) el asentamiento varió en el rango $\underline{70 \pm 20}$ $\underline{\mathrm{mm}}$. Este hecho debe ser atribuido a que los agregados gruesos (naturales y reciclados) fueron saturados durante 24 hs previo a su utilización, de modo que no se produzcan modificaciones en la demanda del agua de mezclado, principalmente por parte de los agregados reciclados. (45020731-I)

(14) Considerando las muestras 3 y 7 puede observarse que, para el hormigón HR-0.50 los valores que adoptan los parámetros $\mathrm{V}$ y Ed resultan similares entre sí, mientras que para el HR-0.60 se observan menores valores en el caso de la muestra 7. Este hecho debe ser atribuido al menor peso específico que presenta dicha muestra de AGR. (45020731-I)

En la sección de Resultados encontramos que las marcas de obligación hacen referencia específica a aspectos propios de la investigación llevada a cabo. En primer lugar, por el tipo de léxico empleado, por ejemplo, palabras o frases que indican lo demostrado con el método, como en El ciclo demostró; también palabras que se refieren a resultados extraídos del instrumento de recolección de datos, como Otras necesidades que se extrajeron del diario de campo llevado durante varias reuniones fueron. Igualmente, encontramos léxico que indica variación, como en, ya que los frutos pueden variar ampliamente en el número de semillas por fruto, otros que indican cantidad, como por ejemplo el asentamiento varió en el rango $70 \pm 20$ $\mathrm{mm}$. Nótese que existen diferencias en cuanto al tipo de léxico empleado por cada disciplina para reportar los resultados. En Educación se privilegia informar sobre lo que demostró el método o la técnica, y se puede prescindir en general de los números (porcentajes). En Botánica se centran en describir variaciones. En esta disciplina no se encontraron suficientes datos numéricos porque los casos de obligación en esta sección del artículo fueron apenas dos. En Ingeniería se da preeminencia al reporte de resultados en forma de cantidades y porcentajes.

Por otro lado, los investigadores de Educación incluían un léxico muy variado, con rasgos de lexicalización para promover cambios deseables es aspectos muy específicos, tal como se ve en el ejemplo (15). 
(15) También señalan la producción agrícola, la alimentación y las artesanías, así como el aprendizaje del castellano como segunda lengua para algunos y como herramienta esencial para la comunicación de todos. La conservación del ambiente está referida tanto al ambiente natural general de la zona amazónica por su importancia global, la protección de la flora, fauna, ríos, bosques, como a la necesidad de resolver problemas específicos como la basura, disposición de excretas, incendios, contaminación de aguas por actividades ilegales, fronteras sin vigilancia y otros. (28820708-E)

El foco de la obligación en este ejemplo se encuentra, por un lado, en el tópico y la localidad geográfica estudiada, y por el otro, en la necesidad de promover cambios deseables en las estrategias que orienten valores de solidaridad y en las estrategias de enseñanza. En el caso de Botánica y de Ingeniería (ejemplos 12,13,14), en la actitud ante el "deber ser" del "conocimiento de la investigación', los autores argumentan las posibles razones por las cuales se produjeron los resultados. Existen señales discursivas que nos permiten identificar los argumentos de los autores, por ejemplo, cuando en Botánica se dice que es conveniente considerar que el número de semillas por frutos representa un mejor estimado reproductivo, y cuando se señala en Ingeniería que este hecho debe ser atribuido a que los agregados gruesos (naturales y reciclados) fueron saturados durante 24 hs previo a su utilización, se está orientando a los lectores/ investigadores sobre la forma en que se deben interpretar los resultados o hallazgos. Sostenemos que en Ingeniería y Botánica los resultados necesitan ser explicados para lograr convencer al experto sobre las razones por las cuales se llegó a los resultados obtenidos. Esto marca una gran diferencia con Educación, porque en esta disciplina generalmente los autores apelan a los textos citados en la investigación de otros como argumento de autoridad.

\subsubsection{En la sección de Conclusiones: ¿qué se debería hacer en la materia y en la} investigación?

Para esta sección se encontró que los verbos, tanto en Educación como en Ingeniería, señalan la obligación en términos de acciones y de atributos, mientras que en el caso de Botánica la necesidad es una acción. Por otra parte, la obligación del 'deber ser' está asociada con la actitud ante la disciplina. Esto en cuanto a:

a. Cómo deben ser complementadas las acciones.

(16) Por ejemplo, las expresiones artísticas como el teatro, la literatura, la pintura o el baile, son básicas para el desarrollo humano y esenciales en los momentos en que la salud está debilitada. Ellas procuran la expresión personal y la expansión del espíritu. Esta acción debe ser complementada con la creación de las "ludotecas rodantes" y mesas de trabajo portátiles, que puedan movilizarse hasta cualquier ambiente del hospital. (28830712-E)

b. Lo que se debe hacer para conocer las necesidades de la población.

(17) Se plantea la necesidad de realizar un censo en el estado para determinar y ubicar la incidencia de condiciones especiales en la población, las demandas por los diferentes tipos de atención, y las posibilidades de responder a su solución en cada municipio. (28830708-E) 
c. Lo que no es deseable o que no requieren las plantas.

(18) Tomando en cuenta que la mayoría de las lianas estudiadas en esta investigación tenían diámetros hasta de $5 \mathrm{~cm}$, existe la posibilidad de que aún presenten ciertos caracteres juveniles y esto influya sobre la presencia de radios heterocelulares con más de una ruta de células marginales. Otra teoría indica que las lianas pueden desarrollar grandes radios ya que no requieren de gran cantidad de tejido de soporte y los radios grandes pueden ayudar en aspectos de flexibilidad y esfuerzo. (30010719-B)

d. Lo que se requiere en la investigación y en la materia.

(19) No obstante, se requieren nuevos estudios morfométricos en un mayor número de géneros y especies de Ingeae y otras Mimosoideae para validar las tendencias encontradas en este trabajo. (30010720-B)

(20) Es necesario contar con resultados experimentales que consideren aspectos no contemplados en este trabajo, tales como: número mayor de machones, muros de otras relaciones de aspecto global, paños de mampostería [...]. (45020733-I)

Para la sección de Conclusiones en Educación, las necesidades son expresadas en términos de acción y de atributos. En el primer caso, nótese que la acción queda evidenciada cuando en el texto (16) el autor señala "la creación de ludotecas rodantes". En este caso el verbo crear indica una acción material concreta. En el ejemplo (17) se indica directamente con la frase nominal 'la necesidad de realizar' algo y también se dan los detalles sobre la circunstancias de la acción, en este caso, la necesidad de responder a las carencias de la población y la necesidad de conocerlas para mejorar la calidad de la enseñanza de esa población. En el ejemplo (18) tomado de Botánica, el verbo requerir señala lo que no se desearía que ocurriera según lo especifica una teoría. Este argumento es utilizado para justificar los resultados obtenidos y para prevenir una posible equivocación en la implementación del método. Llaman la atención los casos (19 y 20), en lo que se señala la necesidad de validar resultados con estudios nuevos y considerar otros aspectos en el mismo estudio. Este acto puede considerarse de modestia por parte de los autores, quienes prefieren no cerrar la investigación. Ello significa que la obligación en estos dos últimos casos tiene el foco en la necesidad de que se siga estudiando el problema y ampliar las categorías de análisis.

En definitiva, las marcas de obligación para cada disciplina y para cada sección del artículo, por un lado, enfatizan la necesidad de que quien sea responsable tiene la obligación de hacer que el evento ocurra; y por el otro, la necesidad de la obligación parece estar más asociada con un plano idealista, o lo que se esperaría que el responsable realizara.

\section{Conclusiones y recomendaciones}

En nuestro estudio encontramos que existe una fuerte tendencia en Educación a usar la modalidad de obligación, ello con un 62.12\% sobre un 26,17\% en Ingeniería y 4,69\% en Botánica. Estos resultados coinciden parcialmente con los encontrados por Beke y Bolívar (2009), cuando al examinar artículos de investigación de las humanidades encontraron, entre otros hallazgos, una fuerte inclinación en Educación por el uso de la modalidad de obligación. Por otro lado, tanto el análisis cuantitativo como el cualitativo de los textos escogidos han permitido evidenciar 
varios aspectos importantes de la modalidad de obligación en las disciplinas. La actitud de los investigadores en las humanidades tiende a evaluar el mundo fuera de la propia investigación. Esto es evidenciado en el mayor número de marcas en la sección de la Introducción y sobre el conocimiento de la materia. Generalmente los usos de la obligación se relacionan con lo deseable en cuanto a las estrategias didácticas empleadas por los docentes, la formación de los docentes, la función de la escuela, el libro de texto escolar como herramienta didáctica, entre otros. La modalidad de obligación en esta disciplina está asociada a lo que sería moralmente correcto para el fortalecimiento más que de la disciplina de la sociedad.

En las ciencias básicas y aplicadas (Botánica e Ingeniería), la evaluación se dirige a la propia investigación. De hecho, existe menos propensión en estas disciplinas a escribir extensas introducciones y marcos teóricos, y centrarse en los aspectos que tienen que ver con el método y los resultados. La función de la obligación en Botánica e Ingeniería se centra en aspectos relacionados con lo deseable en los procedimientos y técnicas a emplearse en los procesos investigativos. Por consiguiente, se enfatizan más los aspectos relacionados con la propia investigación, como el método, los resultados y las conclusiones que se derivan de su estudio. Llama la atención, en la sección de conclusiones del artículo de investigación de estas dos últimas disciplinas, la escritura de argumentos para justificar los hallazgos obtenidos, y sobre los cuales parece haber mayor compromiso en todo el proceso de la investigación.

Adicionalmente, la concepción de investigación entre estas tres disciplinas (Educación, Botánica e Ingeniería) está bastante diferenciada. En Educación el compromiso modal se dirige mayormente en promover cambios a nivel del Sistema Educativo y de la Sociedad, mientras que en Ingeniería y en Botánica con la disciplina y el proceso de investigación mismo. Por otra parte, las diferencias en el empleo de las marcas lingüísticas de la obligación en el artículo de investigación se encuentran principalmente determinadas por: a) la disciplina misma y, b) la sección del artículo. El hecho de que hayamos observado esta variación nos ha permitido ver cómo, desde la obligación, se construye y se reporta el conocimiento, y cómo se establece el diálogo en la academia. El análisis realizado permite confirmar los hallazgos encontrados en otras investigaciones (Swales 2004; Hyland 2005; Bolívar 2006; Beke y Bolívar 2009), en cuanto al papel que desempeña el sistema de la evaluación, y más específicamente el de la modalidad de obligación en las disciplinas y en el artículo de investigación.

Del análisis del "deber ser" en las tres disciplinas se desprenden implicaciones importantes para la producción de artículos de investigación. En primer lugar, la variedad de estructuras lingüísticas de obligación con verbos, nominalizaciones y adjetivos, sitúan al artículo de investigación como un género con una producción retórica compleja. Ello lo hace más difícil de producir, especialmente si se toma en consideración, por un lado, la tradición discursiva y epistemológica de cada disciplina; y por el otro, el nivel de experiencia investigativa de quienes escriben artículos de investigación.

En segundo lugar, las funciones de cada marca lingüística y la sección del artículo de investigación apuntan hacia una variación importante en cuanto a los usos del "deber ser" en las disciplinas. Al respecto, pienso que en la medida en la cual los investigadores de las humanidades y de las ciencias básicas y aplicadas comprendan los esquemas de representación del conocimiento de sus interlocutores acerca del género, del mundo, de los roles que se intercambian y se negocian en la interacción, y de las convenciones que determinan cómo decir y escribir las cosas, podrían escribir artículos más representativos para su comunidad discursiva. 


\section{Notas}

1. Este artículo forma parte de un Trabajo de Grado de Maestría, presentado en la Universidad Central de Venezuela para optar al título de Magister Scientarium en Estudios del Discurso. Agradezco a la profesora Adriana Bolívar sus observaciones y todo su apoyo y colaboración como tutora de este trabajo.

2. Las revistas utilizadas para este estudio fueron la Revista de Pedagogía, Acta Botánica Venezuelica y el Boletín Técnico del Instituto de Materiales y Modelos Estructurales IMME. Todas ellas son publicaciones de la Universidad Central de Venezuela, registradas en el Fondo Nacional de Ciencia, Tecnología e Innovación, FONACIT (www.fonacit.gov.ve).

3. Se consideraron únicamente los artículos publicados para el año 2007, porque para el momento en el que se inició el estudio era el volumen más reciente de las tres revistas escogidas. También, porque se encontraban digitalizados todos los números de ese año en los portales SCIelo y Redalyc. Esto último muy importante para el análisis computarizado de los textos.

4. Los textos pertenecen al CORPUS-EBI.Briceño (2009) y forma parte de un corpus mayor denominado CORDA-UCV creado con el Proyecto de Grupo titulado "Discurso e investigación: la escritura de textos científicos y humanísticos” de número PG-07-7830-2009/1 del CDCH-UCV.

\section{Bibliografía}

Bazerman, Charles. 1988. Shaping written knowledge: the genre and activity the experimental article in science. Madison, WI: University Wisconsin Press.

Beke, Rebecca. 2008. "El discurso académico: la atribución del conocimiento en la investigación educativa”. Núcleo. 25: 13-35.

2011. Las voces de los otros en el discurso académico. Caracas: Comisión de Estudios de Postgrado. Facultad de Humanidades y Educación. Universidad Central de Venezuela.

Bhatia, Vijay. 1993. Analysing genre: Language use in profesional settings. Harlow: Longman.

Biber, Douglas, Susan Conrad y Randi Reppen. 1998. Corpus linguistics: investigating language structure and use. Cambridge: Cambridge University Press.

Blanco, Carlos Eduardo. 2007. Revista de Pedagogía. 28 (81-82-83). Caracas: Universidad Central de Venezuela. ISSN 0798-9792.

Bolívar, Adriana. 1996. Los resúmenes para conferencias en lingüística aplicada en América Latina: estructura e interacción. I Coloquio Internacional de Lingüística Aplicada, Linguística del Texto. Producción de textos. Universidad Nacional de Córdoba, 19 y 20 de septiembre.

1997. "La pragmática lingüística de los resúmenes de investigación para congresos". Boletín de Lingüística. 12 (13): 153-173.

1999. “Las metafunciones de la cláusula en español”. Lingua Americana. III (4): 48-66. 2001. "The negotiation of evaluation in written text". En: Mike Scott y Geoff Thompson (Eds.). 129-158.

2004. "Análisis crítico del discurso de los académicos". Signos. 37 (55): 7-18.

2005. Discurso e interacción en el texto escrito. Caracas: Universidad Central de Venezuela. 
2006. "La función de la evaluación en artículos y ensayos humanísticos". En: Gille Johan y Fernando Wachtmeister Bermúdez (Eds.). 11-136.

2008. "El informe de arbitraje como género discursivo en la dinámica de la investigación”. Revista Latinoamericana de Estudios del Discurso. 8 (1): 41-64.

2011. "The interface between grammar, pragmatics and discourse in peer reviews of research articles in Spanish". Ponencia presentada en la $12^{\text {th }}$ International Pragmatics Conference, Manchester, U.K, 3-8 July.

Bolívar, Adriana y Francisco Bolet. 2011. La introducción y la conclusión en el artículo de investigación. En: Adriana Bolívar y Rebecca Beke (Eds.). 93-129.

Bolívar, Adriana y Martha Shiro. 2005. "La modalidad epistémica y deóntica en el discurso académico escrito”. Congreso Internacional de la ALFAL, Monterrey, México, 17-20 de octubre.

Bolívar, Adriana y Rebecca Beke. 2009. Certainty and commitment in the construction of academic knowledge in the humanities. En: Eija Suomela-Salmi y Fred Dervin (Eds.). 33-48.

2011. Lectura y escritura para la investigación. Caracas: Consejo de Desarrollo Científico y Humanístico. Universidad Central de Venezuela.

Bolívar, Adriana, Rebecca Beke y Martha Shiro. 2010. "Las marcas lingüísticas del posicionamiento en las disciplinas: estructuras, voces y perspectivas discursivas". En: Giovanni Parodi (Ed.). 95-125.

Briceño Velazco, Yosely. 2010. El discurso del “deber ser” en artículos de investigación de educación, botánica e ingeniería. Trabajo de grado de Magister en Estudios del Discurso: Universidad Central de Venezuela.

Coulthard, Michael (Ed.). 1994. Advances in written text analysis. London: Routledge.

Chafe, Wallace. 1986. "Evidentiality in English conversation and academic writing". En: Wallace Chafe y Johanna Nichols (Eds.). 261-72.

Chafe, Wallace y Johanna Nichols (Eds.). 1986. Evidentiality: the linguistics coding of epistemology. Norwood, NJ: Ablex.

Ferrari, Laura. 2003. "Modalidad epistémica y grados de certeza en los artículos de investigación". Ponencia presentada en el III Congreso de Lenguas del Mercosur: De la teoría a la Praxis de las Lenguas, Universidad Nacional del Nordeste, Resistencia Chaco, Argentina.

Ferrari, Laura y Susana Gallardo. 2006. "Estudio diacrónico de la evaluación en las introducciones de artículos científicos de medicina”. Signos. 39 (61): 161-180.

Flowerdew, John (Ed.). 2002. Academic discourse. London: Logman.

García Negroni, María Marta. 2008. "Subjetividad y discurso científico-académico. Acerca de algunas manifestaciones de la subjetividad en el artículo de investigación en español". Signos. 41 (66): 5-31.

Gille Johan y Fernando Wachtmeister Bermúdez (Eds.) 2006. Discurso, interacción e identidad. Homenaje a Lars Fant. Stockholm: Stockholm Universiteit. 
Gutiérrez, Rosa María. 2008. "El género manual en las disciplinas académicas: una caracterización desde el sistema de la obligación”. Signos. 41 (67): 177-202.

Halliday, Michael. 1994. An introduction to functional grammar. Second Edition. London: Edward Arnold.

Harvey, Ana María (Comp.). 2005a. En torno al discurso. Contribuciones de América Latina. Santiago: Ediciones Universidad Católica de Chile.

2005b. "Manifestaciones evaluativas en la ciencia como discurso. Un estudio comparativo”. En: Ana María Harvey (Comp.). 94-110.

Hunston, Susan. 1994. "Evaluation and organization in a sample of written academic discourse". En: Michael Coulthard (Ed.). 191-218.

2001. "Evaluation and plans of discourse: Status and value in persuasive texts". En: Susan Hunston y Geoff Thompson (Eds.). 176-206.

2002. Corpora in Applied Linguistics. Cambridge: Cambridge University Press.

Hunston, Susan y Geoff Thompson (Eds.). 2000. Evaluation in text. Authorial stance and the construction of discourse. Oxford: Oxford University Press.

2006. System and corpus: Exploring connections. London: Equinox.

Hyland, Ken. 1999. "Academic attribution: citation and the construction of disciplinary knowlwdge". Applied Linguistics. 20 (3): 341-367.

2000. Disciplinary discourses: Social interactions in academic writing. Harlow: Logman.

2002. "Activity and evaluation: reporting practices in academic writing". En: John Flowerdew (Ed.). 115-130.

2004. "Disciplinary interactions: metadiscourse in L2 postgraduate writing". Journal of Second Language Writing. 13: 113-155.

2005. "Stance and engagement: A model of interaction in academic discourse". Discourse Studies. 7 (2): 173-192.

Hyland, Ken y Giuliana Diani (Eds.). 2009. Academic evaluation. Review genres in university settings. Londres: Palgrave Macmillan.

Marinilli, Angelo. 2007. Boletín Técnico del Instituto de Materiales y Modelos Estructurales. 45 (1-2-3). Caracas: Universidad Central de Venezuela. ISSN 0376-723X.

Martin, James y Peter White. 2005. The language of Evaluation. Appraisal in English. Londres/New York: Palgrave Macmillan.

Palmer, Frank Robert. 2001. Mood and modality. Cambridge: Cambridge University Press.

Paltridge, Brian. 1994. "Genre analysis and the identification of textual boundaries". Applied Linguistics. 15 (3): 288-299.

Parodi, Giovanni. 2005. "Discurso especializado y lingüística de corpus: hacia el desarrollo de una competencia psicolingüística”. Boletín de Lingüística. 17 (23): 61-88. 
2006. "Discurso especializado y lengua escrita: foco y variación". Estudios Filológicos. 41: $165-204$.

2007. "El discurso especializado escrito en el ámbito universitario y profesional: constitución de un corpus de estudio". Signos. 40 (63): 147-178.

2010. "Alfabetización académica y profesional en el siglo XXI: leer y escribir desde las disciplinas". Santiago: Planeta.

Pérez-Cortéz, Silvia. 2007. Acta Botánica Venezuelica. 30 (1-2). Caracas: Universidad Central de Venezuela. ISSN 0084-5906.

Samraj, Betty. 2002. "Introduction in research articles: variations across disciplines". English for Specific Purposes. 21: 1-17.

Sinclair, John. 2003. Reading concordances. Harlow: Pearson Education. 2004. Trust the text: Language, corpus and discourse. London: Routledge.

Simpson, Rita y John Swales (Eds.). 2001a. Corpus Linguistics in North America. Selections from the 1999 Symposium. Ann Arbor: The University of Michigan Press.

2001b. "Introduction. North American perspectives on Corpus Linguistics at the Millennium". En: Rita Simpson y John Swales (Eds.). 1-14.

Scott, Mike y Geoff Thopmson (Eds.). 2001. Patterns of texts in honour of Michael Hoey. Amsterdan: John Benjamins.

Stubbs, Michael. 1996. Text and Corpus Analysis. Computer assisted Studies of Language and Culture. Oxford: Blackwell.

2006. "Corpus analysis: The state of the art and three types of unanswered questions". En: Susan Hunston y Geoff Thompson (Eds.). 15-36.

Suomela-Salmi, Eija y Fred Dervin (Eds.). 2009. Cross linguistic and cross cultural perspectives on Academic Discourse. Amsterdam: John Benjamins.

Swales, John. 1990. Genre Analysis. New York: Cambridge University Press.

2004. Research genres. Explorations and applications. Cambridge: Cambridge University Press.

Thompson, Geoff. 1996. Introducing functional grammar. London: Arnold.

Tse, Polly y Ken Hyland. 2009. "Discipline and gender". En: Ken Hyland y Giuliana Diani (Eds.). 95-121.

Venegas, René. 2006. "La similitud léxico-semántica en artículos de investigación científica en español: Una aproximación desde el análisis semántico latente”. Signos. 39 (60): 75-106. 
